Article

\title{
Live Biosensors for Ultrahigh-Throughput Screening of Antimicrobial Activity against Gram-Negative Bacteria
}

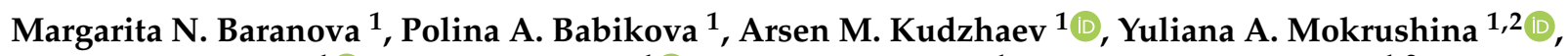 \\ Olga A. Belozerova ${ }^{1} \oplus$, Maxim A. Yunin ${ }^{1}{ }^{\circledR}$, Sergey Kovalchuk ${ }^{1}$, Alexander G. Gabibov ${ }^{1,2, *}$, Ivan V. Smirnov ${ }^{1,2, *}$ \\ and Stanislav $S$. Terekhov $1,2, *$ (D) \\ 1 Shemyakin-Ovchinnikov Institute of Bioorganic Chemistry of the Russian Academy of Sciences, \\ 117997 Moscow, Russia; baranova@ibch.ru (M.N.B.); babikova.pa@phystech.edu (P.A.B.); \\ kudzhaev_arsen@mail.ru (A.M.K.); yuliana256@mail.ru (Y.A.M.); o.belozyorova@gmail.com (O.A.B.); \\ yuninma@gmail.com (M.A.Y.); xerx222@gmail.com (S.K.) \\ 2 Department of Chemistry, Lomonosov Moscow State University, 119991 Moscow, Russia \\ * Correspondence: gabibov@ibch.ru (A.G.G.); smirnov@ibch.ru (I.V.S.); sterekhoff@gmail.com (S.S.T.)
}

\section{check for}

updates

Citation: Baranova, M.N.; Babikova, P.A.; Kudzhaev, A.M.; Mokrushina, Y.A.; Belozerova, O.A.; Yunin, M.A.; Kovalchuk, S.; Gabibov, A.G.; Smirnov, I.V.; Terekhov, S.S. Live Biosensors for Ultrahigh-Throughput Screening of Antimicrobial Activity against Gram-Negative Bacteria. Antibiotics 2021, 10, 1161. https:// doi.org/10.3390/antibiotics10101161

Academic Editor: Helena Felgueiras

Received: 3 September 2021

Accepted: 22 September 2021

Published: 24 September 2021

Publisher's Note: MDPI stays neutral with regard to jurisdictional claims in published maps and institutional affiliations.

Copyright: (c) 2021 by the authors. Licensee MDPI, Basel, Switzerland. This article is an open access article distributed under the terms and conditions of the Creative Commons Attribution (CC BY) license (https:// creativecommons.org/licenses/by/ $4.0 /)$.

\begin{abstract}
Gram-negative pathogens represent an urgent threat due to their intrinsic and acquired antibiotic resistance. Many recent drug candidates display prominent antimicrobial activity against Gram-positive bacteria being inefficient against Gram-negative pathogens. Ultrahigh-throughput, microfluidics-based screening techniques represent a new paradigm for deep profiling of antibacterial activity and antibiotic discovery. A key stage of this technology is based on single-cell cocultivation of microbiome biodiversity together with reporter fluorescent pathogen in emulsion, followed by the selection of reporter-free droplets using fluorescence-activated cell sorting. Here, a panel of reporter strains of Gram-negative bacteria Escherichia coli was developed to provide live biosensors for precise monitoring of antimicrobial activity. We optimized cell morphology, fluorescent protein, and selected the most efficient promoters for stable, homogeneous, high-level production of green fluorescent protein (GFP) in E. coli. Two alternative strategies based on highly efficient constitutive promoter pJ23119 or T7 promoter leakage enabled sensitive fluorescent detection of bacterial growth and killing. The developed live biosensors were applied for isolating potent E. coli-killing Paenibacillus polymyxa P4 strain by the ultrahigh-throughput screening of soil microbiome. The multi-omics approach revealed antibiotic colistin (polymyxin E) and its biosynthetic gene cluster, mediating antibiotic activity. Live biosensors may be efficiently implemented for antibiotic/probiotic discovery, environmental monitoring, and synthetic biology.
\end{abstract}

Keywords: ultrahigh-throughput screening; live biosensors; antibiotic discovery; gram-negative pathogens; microfluidic droplet cocultivation; efficient promoters; polymyxins; colistin biosynthetic gene cluster; single cell; multi-omics

\section{Introduction}

Global use of antimicrobials provokes intensive antimicrobial resistance (AMR) selection. AMR represents a threat to sustainable development, leading to 11 million deaths annually [1,2]. The recent COVID-19 pandemic resulted in a significant increase in antibiotic sales [3] and extensive antibiotic use without proper clinical indication [4]. Hence, the overuse of antibiotics lays the foundations for further resistome propagation and multi-resistance evolution. Further deterioration in this field threatens the emergence of epidemics caused by multiresistant pathogens and their subsequent persistence in the population under selection pressure.

Gram-negative pathogens represent a particularly dangerous cohort, including three of five urgent threats highlighted by the Centers for Disease Control and Prevention (CDC) [5]. New antibiotics targeting resistant Gram-negatives have been approved, but most of them belong to existing classes of antibiotics, and resistance to them has already 
emerged [6]. Gram-negative bacteria have an outer membrane, a protective and unique feature that distinguishes them from Gram-positive bacteria. This shield provides an efficient barrier for a vast variety of antimicrobials. Together with acquired resistance mechanisms, like mutations in chromosomal genes or mobile genetic elements carrying resistance genes, this provides a challenge to medication, often unresolvable [7].

Despite the urgent antibiotic rediscovery problem [8], classical antibiotic-producing species still provide a source for new drug candidates [9]. However, exotic microbial communities represent a more promising reservoir for the isolation of new antibiotics [10]. Recently, we developed an ultrahigh-throughput microfluidic platform for biodiversity screening [11]. This technology is based on single-cell cultivation of microorganisms in isolated microcompartments of double water-in-oil-in-water emulsion with subsequent isolating phenotypes of interest by fluorescence-activated cell sorting (FACS). More than 10,000 single bacterial clones may be screened for antibiotic activity in a second to isolate the most efficient antibiotic producers [12] or resistant strains [13]. This productivity enables deep functional profiling of microbiota communities [12] and the discovery of new molecular mechanisms of resistance [14]. The critical step of this technique is a coencapsulation of a highly fluorescent reporter GFP-producing bacterial strain together with single cells from the microbiome followed by their cocultivation in droplet compartments. Efficient bacterial killers are subsequently selected with FACS by a low GFP fluorescence level in a minor subpopulation of droplets. Previously, this platform was implemented for deep profiling of anti-S. aureus activity [11-13]. In this study, cell morphology, fluorescent protein nature, and promoter efficiency were optimized to adopt this strategy for extensive anti-Gram-negative screening based on model bacteria E. coli. Common laboratory E. coli strains, including Rosetta, BL21(DE3), TG1, XL-1, and SHuffle T7, were investigated to maximize GFP fluorescence and homogeneity. Two different green fluorescent proteins, i.e., TagGFP2 [15] and sfGFP [16], were examined as reporters in live biosensors. Moreover, the efficiency of GFP production under the control of different promoters was compared, including highly efficient constitutive E. coli promoters, i.e., pEm7 [17], pglpT [18], pJ23119 [19], OXB20 [20], and leaking $\mathrm{T7}$ promoter.

We obtained that both highly efficient constitutive promoter $\mathrm{pJ} 23119$ and T7 promoter leakage enable sensitive fluorescent detection of bacterial growth and killing. Live biosensor based on BL21(DE3) strain, producing sfGFP via leaking T7 promoter, outperformed pJ23119 in terms of fluorescence level, while the fluorescence distribution of T7-based reporters was higher than pJ23119. Finally, pJ23119-sfGFP BL21(DE3) reporter cells were applied for a proof-of-concept soil microbiome screening. In a single round of screening, a potent E. coli-killing Paenibacillus polymyxa P4 strain was isolated and analyzed by complex multi-omics strategy, including activity-based metabolomics and genomics. Colistin (polymyxin E) was determined as a key metabolite mediating anti-Gram-negative antibiotic activity. The identified biosynthetic gene cluster (BGC) of colistin displayed close similarity to BGCs of polymyxin A [21], D-Dab ${ }_{3}$-polymyxin B [22], and polymyxin E [23]. However, it did not contain the epimerization domain in module 3 , unlike all previously published BGCs of polymyxins. We believe that the developed live biosensors may be efficiently implemented for ultrahigh-throughput screening of antimicrobial activity against gram-negative bacteria for antibiotic/probiotic discovery, environmental monitoring, and synthetic biology.

\section{Results}

\subsection{General Requirements for Live Biosensors Applied in Ultrahigh-Throughput Screening}

Ultrahigh-throughput screening of antimicrobial activity is based on a single-cell encapsulation of microbiome representatives together with fluorescent protein-producing reporter bacteria followed by isolation of droplets with inhibited growth of reporter bacteria using FACS (Figure 1). 


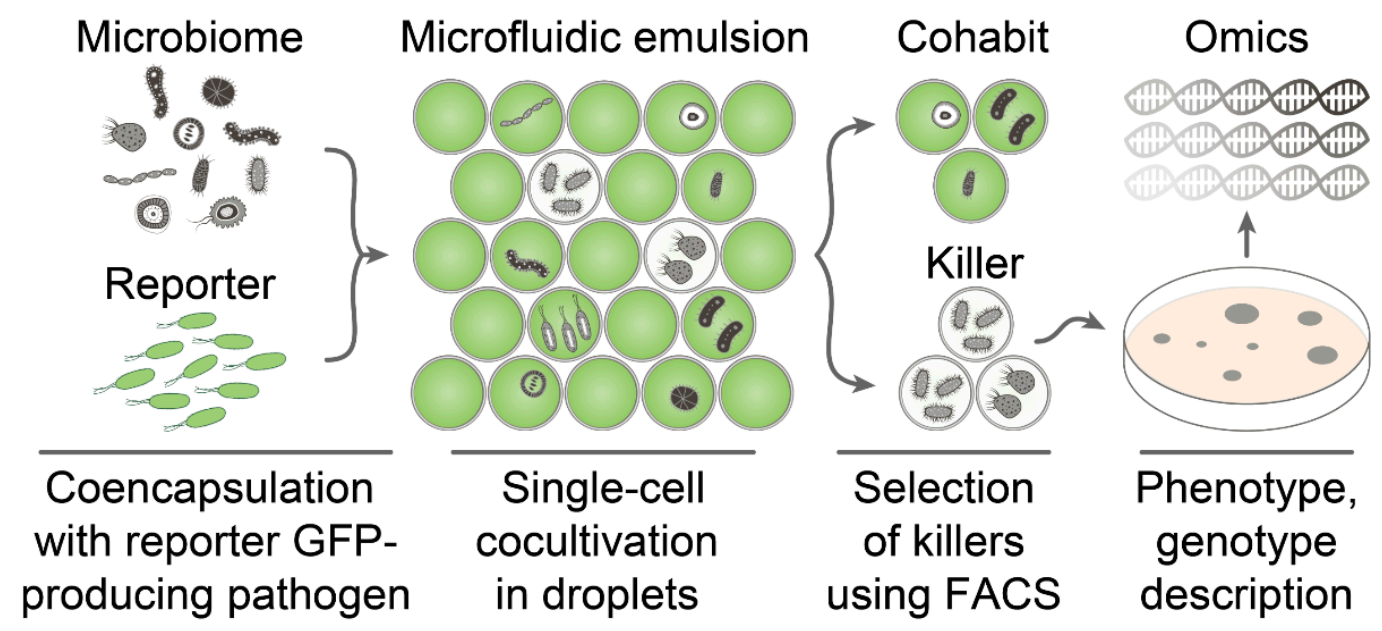

Figure 1. The general pipeline of ultrahigh-throughput screening of antimicrobial activity. The bacterial community is encapsulated with reporter GFP-producing pathogen in biocompatible droplets of microfluidic double water-in-oil-in-water emulsion. Cocultivation of bacterial community with reporter bacteria in droplets results in two distinct populations containing bacterial cohabits and killers. The latter is selected by a low level of GFP fluorescence by FACS. The selected droplets are plated on agar to regenerate culturable killers analyzed by activity-guided metabolomics and genomics. Detailed phenotype and genotype description enable identifying antibiotics and their biosynthetic gene clusters.

The critical component of this platform is a reporter pathogen strain. It must follow certain criteria, essential for efficient screening: (1) Cells must have regular morphology; aggregates or big non-uniform cells are undesirable; (2) production of fluorescent protein should be constitutive and homogeneous in population; and (3) a high level of cell culture fluorescence is required for precise detection of antimicrobial activity. A model Gram-negative bacteria E. coli was optimized following these criteria to provide efficient, live biosensors for ultrahigh-throughput screening and sensitive antimicrobial activity detection.

\subsection{Selection of GFP-Producing Strain}

Cell morphology plays an important role for reporter strain selection since cell aggregate clot microfluidic channels, while big and irregular cells tend to sediment in fluidics. Moreover, cell aggregates and non-uniformity may influence regular droplet occupancy. Hence, common laboratory E. coli strains including Rosetta (DE3), BL21(DE3), TG1, XL-1, and SHuffle T7 were transformed with pYTK047 plasmid for constitutive production of GFP [24], and cell morphology was visualized by fluorescence microscopy (Figure 2).

BL21(DE3) and TG1 strains of E. coli were suitable as a template for the creation of live biosensors since they provided homogeneous cell cultures with a high level of cell fluorescence. Other strains were inappropriate as a reporter. Rosetta (DE3) had a mediocre fluorescence. Xl-1 had a stretched morphology with a high number of odd rod cells. SHuffle T7 formed cell aggregates. Further, E. coli BL21(DE3) was used for live biosensors' engineering.

\subsection{Stable and Homogeneous Production of GFP in E. coli}

Different fluorescent proteins and promoters were compared to maximize the fluorescence of live E. coli culture (Figure 3).

While TagGFP2 was declared non-toxic to the host [15], we observed dramatically decreased fluorescence of cell cultures in E. coli transformed with plasmids under control of T7 promoter compared with the same constructs coding sfGFP fluorescent protein (Figure 4A).

Moreover, TagGFP2-producing plasmids based on T7 promoter tend to be lost under induction, resulting in a highly heterogeneous population with varying fluorescence of colonies (Figure 4B). Hence, sfGFP was used as a reporter fluorescent protein. 


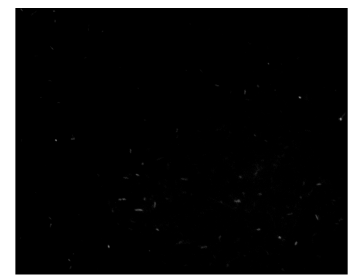

Rosetta (DE3)

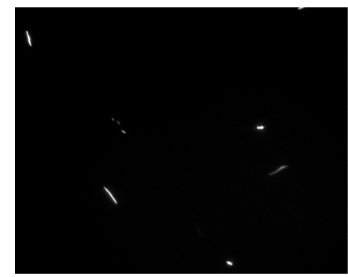

$\mathrm{XL}-1$

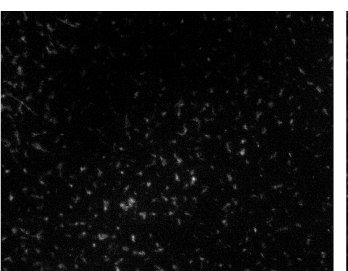

BL21(DE3)

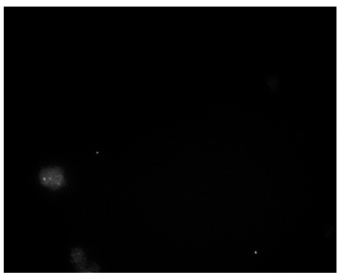

SHuffle T7

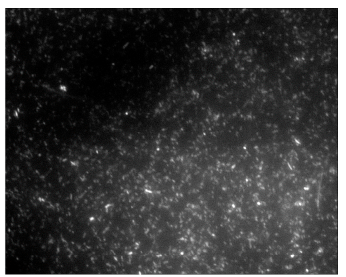

TG1

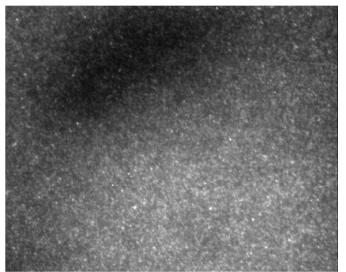

S. aureus

Figure 2. Cell morphology of different $E$. coli strains visualized by fluorescence microscopy. GFPproducing reporter $S$. aureus cells were used as a control.

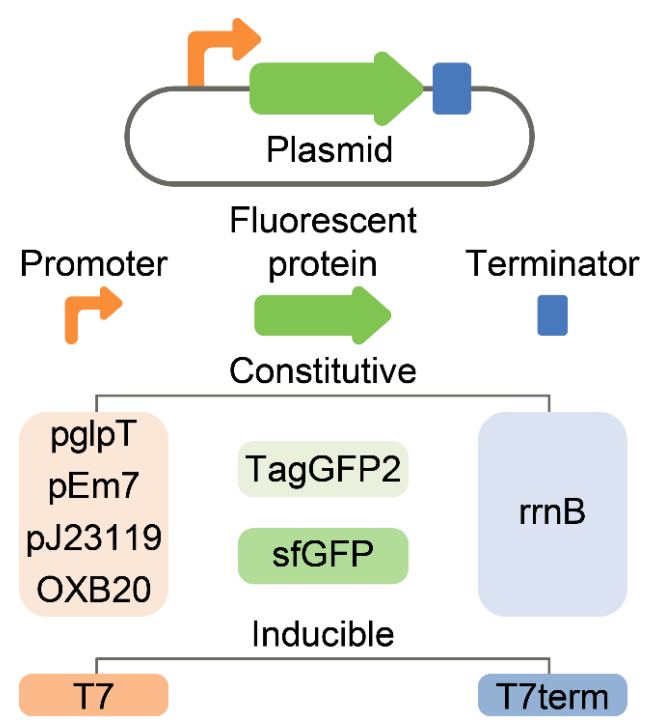

Figure 3. A panel of plasmids that were used for the optimization of E. coli-based live biosensors. All genetic constructs were constructed on the same high-copy number template vector. Two different fluorescent proteins, i.e., TagGFP2 and sfGFP, were used. Constitutive (pglpT, pEm7, pj23119, and OXB20) and inducible (T7) promoters were compared in terms of efficiency, homogeneity of cell fluorescence, and stability.

The efficiencies of different promoters were compared to maximize GFP fluorescence in E. coli. Brain Heart Infusion (BHI) was used as a basal medium since it enables culturing a variety of microorganisms, which is preferred for subsequent ultrahigh-throughput screening of antimicrobial activity. Surprisingly, efficient T7 leakage was observed in E. coli BL21(DE3) (Figure 5A). Highly efficient constitutive E. coli promoters, i.e., pEm7, pglpT, pJ23119, and OXB20, were compared with leaking T7 in agar plates (Figure 5A), culture medium (Figure 5B), and flow cytometry (Figure 5C). Leaking T7 results in 2.3-8.9 times higher fluorescence of bacterial cultures in comparison with constitutive E. coli promoters. The pJ23119 was the strongest constitutive promoter that enabled stable and homogeneous fluorescence of cell cultures, similar to GFP-producing reporter $S$. aureus cells used previously (Figure 5B). While live biosensors based on leaking T7 promoter outperformed pJ23119 in cell cultures, the cell fluorescence distribution of T7-based reporters was higher (Figure 5C). The fluorescence levels of cell cultures were 1.3-1.6 times higher with the TB medium. However, we suggest that $\mathrm{BHI}$ is more preferred to maintain broad biodiversity in emulsion culture. 

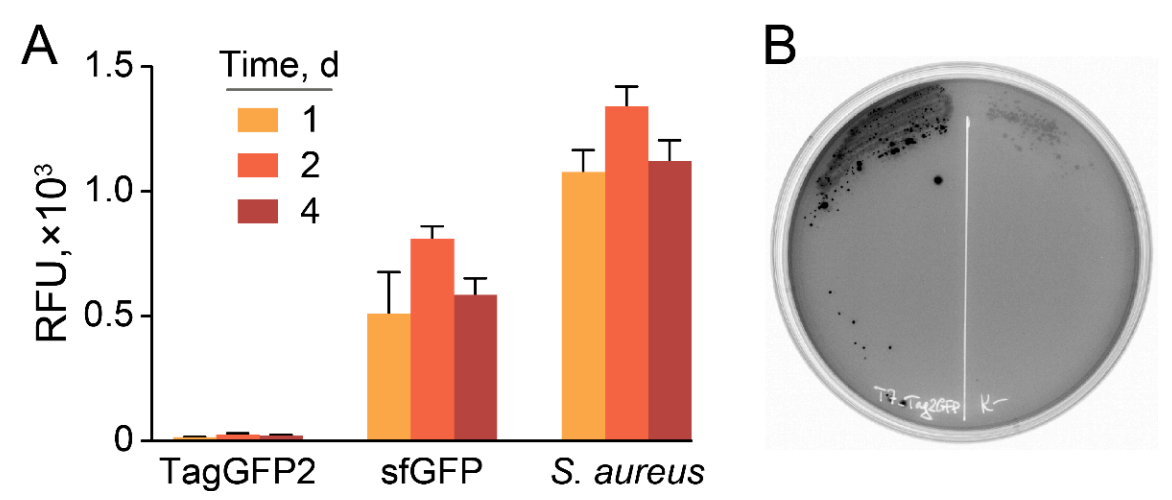

Figure 4. Hyperproduction of TagGFP2 fluorescent protein results in plasmid loss. (A) Fluorescence of cell cultures transformed with synonymous plasmids encoding TagGFP2 and sfGFP fluorescent proteins under the control of T7 promoter, induced by the addition of $1 \mathrm{mM}$ IPTG. GFP-producing reporter $S$. aureus cells were used as a control. Data represent the mean of three biological replicates \pm SD. (B) A representative plate with E. coli-producing TagGFP2 under control of T7 promoter, induced by $1 \mathrm{mM}$ IPTG.

A

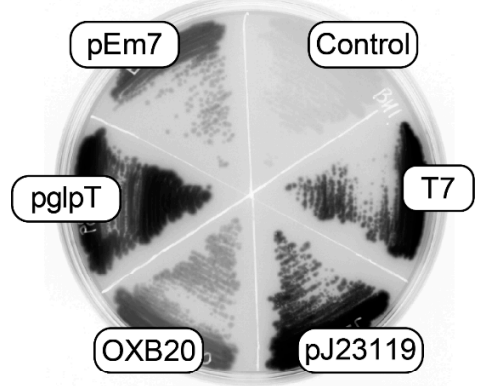

C

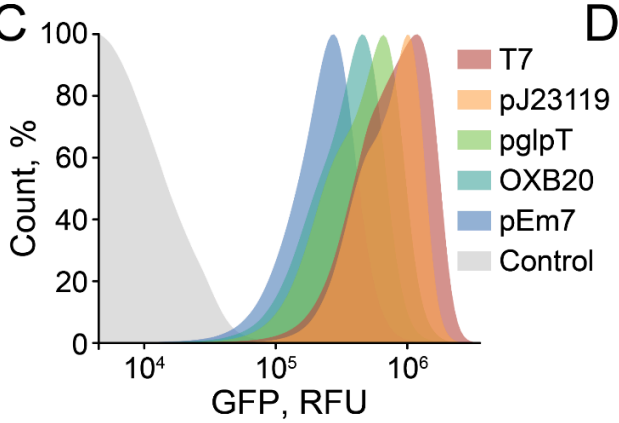

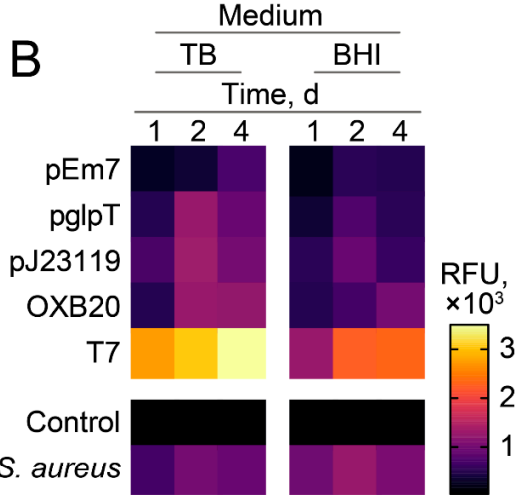

D

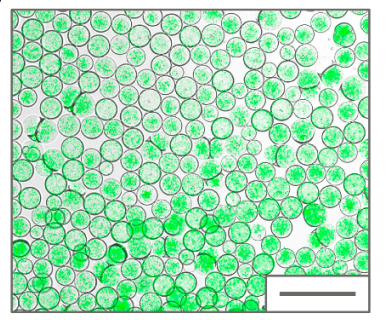

Figure 5. Efficacy of different promoters for constitutive and homogeneous production of GFP in E. coli. (A) A representative BHI-agar plate with E. coli, producing sfGFP under control of various promoters. Constitutive E. coli promoters, i.e., pEm7, pglpT, pJ23119, and OXB20, were compared with leaking T7. E. coli cells transformed with empty vector without sfGFP insert were used as a negative control. (B) Fluorescence of bacterial cultures obtained after 1, 2, and 4 days of cultivation in TB or BHI medium. Relative fluorescence units (RFU) are presented as a heatmap indicating bulk fluorescence of cell cultures. GFP-producing reporter $S$. aureus cells were used as a positive control. Data represent the mean of three biological replicates. (C) Flow cytometry of bacterial cultures producing sfGFP obtained after 2 days of cultivation in BHI medium. (D) Live biosensors producing sfGFP under control of pJ23119 promoter after 1 day of cultivation in droplets of microfluidic double water-in-oil-in-water emulsion. Scale bar: $100 \mu \mathrm{m}$.

Hence, we propose that alternative strategies based on highly efficient constitutive promoter pJ23119 or T7 promoter leakage enabled sensitive fluorescent detection of bacterial growth and killing. Live biosensors producing sfGFP under control of pJ23119 promoter 
were encapsulated in biocompatible droplets of microfluidic double water-in-oil-in-water emulsion with occupancy of $\sim 5 E$. coli cells per droplet. Cultivation of reporters in emulsion resulted in efficient bacterial growth, providing highly fluorescent droplet compartments (Figure 5D) suitable for ultrahigh-throughput screening of antimicrobial activity.

\subsection{Ultrahigh-Throughput Screening of Antimicrobial Activity}

A model ultrahigh-throughput screening of antimicrobial activity was performed to demonstrate the efficiency of the developed live biosensors. Soil microbiome isolated in the Moscow region was used as a source of bacterial biodiversity. GFP-producing live biosensors were coencapsulated with $\sim 10^{6}$ soil microbiome bacteria, followed by cocultivation, selection, and regeneration of culturable anti-E. coli bacteria. Potent E. coli-killing Paenibacillus polymyxa P4 strain was isolated in one round of selection. Metabolomic analysis revealed that polymyxin E (colistin) is the major secondary metabolite active against Gram-negative bacteria. Polymyxins represent closely related lipopeptide antibiotics having a high number of positively charged 2,4-diaminobutyric acid (Dab) residues and hydrophobic residues of Leu and Phe (Figure 6A). Similar to previously identified Paenibacillus alvei B-LR [23], P. polymyxa $\mathrm{P} 4$ produced two analogous polymyxins $\mathrm{E}_{1}$ and $\mathrm{E}_{2}$ that differ by 6-methyloctanoic acid and 6-methylheptanoic acid moieties, respectively (Figure 6B).

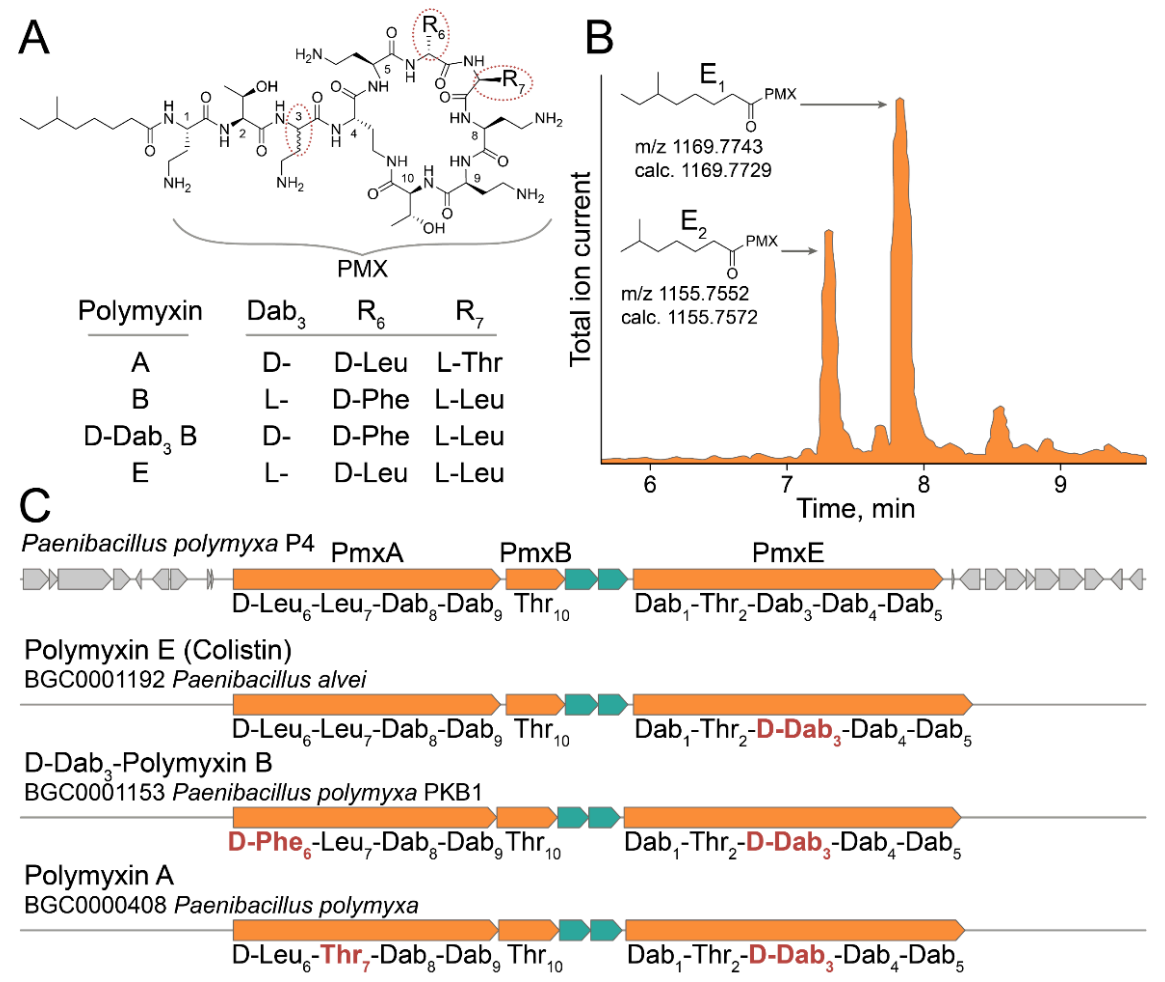

Figure 6. Multi-omic characterization of Paenibacillus polymyxa P4 strain selected by ultrahighthroughput screening of anti-Gram-negative antibiotic activity using the developed live biosensor. (A) Polymyxin E (colistin) was identified as a major secondary metabolite of P. polymyxa P4 active against Gram-negative bacteria. The chemical structures of polymyxin E and related polymyxins A, $\mathrm{B}$, and D-Dab 3 B produced by distinct Paenibacillus are presented. PMX - colistin peptide backbone. (B) LC-MS chromatogram of active fraction of P. polymyxa P4 metabolites. Specific peaks of polymyxin $\mathrm{E}_{1}$ and polymyxin $\mathrm{E}_{2}$ are presented with their experimental and calculated $[\mathrm{M}+\mathrm{H}]^{+}$molecular ion masses. (C) BGC of polymyxin E identified in the genome of P. polymyxa P4. Core NRPSs (PmxA, PmxB, and PmxE) and ABC transporters are colored with orange and aquamarine, respectively. Predicted amino acid specificity of NRPS modules are presented. Related BGCs of polymyxin A [21], D-Dab 3 -polymyxin B [22], and polymyxin E [23] are presented with their predicted modular specificities. Distinct modular specificities are highlighted with red. 
Whole genome sequencing of P. polymyxa $\mathrm{P} 4$ enabled identifying the biosynthetic gene cluster (BGC) of polymyxin E (Figure 6C). P. polymyxa P4 polymyxin E BGC has the same architecture as all polymyxin BGCs mediating the production of polymyxin A [21], D-Dab3-polymyxin B [22], and polymyxin E [23] identified previously. Polymyxin E BGC

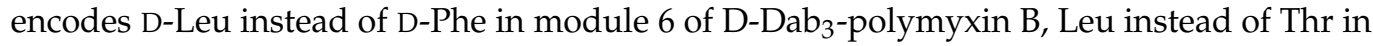
module 7 of polymyxin A, and Dab instead of D-Dab in module 3 of all known polymyxins. While modules 6 and 7 encode amino acid residues varying between polymyxins, module 3 does not have an epimerization domain. Hence, we predicted that the identified polymyxin E produced by P. polymyxa P4 has natural L-stereochemistry. It was recently shown that the epimerization domain of module 3 may be functional, at least in the case of $P$. polymyxa PKB1 strain producing D-Dab 3 -polymyxin B [22]. Therefore, the stereochemistry of Dab $\mathrm{Da}_{3}$ is questionable for some polymyxins. However, it is unambiguous for the identified polymyxin E produced by P. polymyxa P4.

\section{Discussion}

Estimates indicate that antibiotics not yet discovered are likely to be produced at frequencies as low as $\leq 1$ in $10^{7}$ in fermentation broths from random actinomycetes [25]. Hence, deep profiling of antimicrobial activity of microbiomes on a single-cell level provides a new perspective to antibiotic discovery. The miniaturization of antibiotic activity assays is an essential step forward to the next-generation screening platforms. Microfluidic technologies enable single-cell bacterial culturing [26], enzymatic activity screening [27], and antimicrobial activity profiling [28]. Biocompatible droplet microcompartments allow transitioning from classical 2D culture on the surface of agar plates to $3 \mathrm{D}$ emulsion culture. This transition results in a dramatic increase in explored diversity since $\sim 10^{6}-10^{9}$ unique bacterial clones may be cultured in $1 \mathrm{~mL}$ of $100-10 \mu \mathrm{m}$ emulsion droplets instead of $\sim 10^{2}-10^{3}$ clones on a single agar plate.

Here, we described how ultrahigh-throughput technologies could be applied for antimicrobial activity screening against Gram-negative bacteria. Live biosensors based on engineered GFP-producing E. coli enable detecting bacterial antagonism of individual bacterial clones cultured in droplet microcompartments. Different $E$. coli strains, GFPs, and promoters were tested to optimize cell morphology and maximize cell fluorescence. T7 promoter leakage enables sensitive fluorescent detection of bacterial growth and killing regardless of IPTG or lactose induction using common laboratory strain BL21(DE3) and high-copy number plasmid. Highly efficient constitutive promoter pJ23119 may be an alternative with a slightly reduced fluorescence level but increased homogeneity of fluorescence. Finally, the efficiency of engineered live biosensors was illustrated by the ultrahigh-throughput screening of E. coli-killing bacteria. P. polymyxa $\mathrm{P} 4$ strain producing potent anti-Gram-negative antibiotic polymyxin $\mathrm{E}$ was isolated using a single round of selection. The biosynthetic gene cluster (BGC) of polymyxin $\mathrm{E}$ has the same architecture as polymyxin $\mathrm{A}$, D-Dab 3 -polymyxin $\mathrm{B}$, and polymyxin E BGCs identified previously. The unique feature of the identified polymyxin E BGC is that its module 3 does not have an epimerization domain confirming the L-stereochemistry of $\mathrm{Dab}_{3}$ residue.

Engineered live biosensors provide a simple, efficient, and highly sensitive tool for precise monitoring of antimicrobial activity. Basic principles of their construction may be transferred to different Gram-negative bacteria. The implementation of ultrahighthroughput technologies in antibiotic discovery enables deep profiling of antimicrobial activity, accelerating hit identification, and expanding biodiversity coverage. While a number of problems regarding the cultivation of unculturable microorganisms should be resolved to amplify the power of this technique, microfluidic droplet platforms already outperform classical cultivation strategies in some applications [26]. Another option is a search for specific potentiating agents targeting antibiotic resistance. In this case, using an AMR reporter strain supplemented with a conventional antimicrobial may be a target if the antibiotic-producing microorganism is resistant. It may be achieved using naturally resistant bacterial killers like fungi or engineered strains having improved efflux or mu- 
tation providing resistance. The application field of live biosensors is not restricted to microfluidics-based technologies like ultrahigh-throughput screening. Live biosensors may provide sensitive detection of antimicrobial activity in a broad sense, including such applications as routine antibiotic/probiotic screening, pollution monitoring, detection of antibiotic contamination, and more sophisticated fields based on synthetic biology principles.

\section{Materials and Methods}

Genetic constructs and strains. All genetic constructs were based on high-copy vector plasmid PURExpress Control DHFR Plasmid (NEB, Ipswich, MA, USA). Multiple cloning sites including XbaI, XhoI, and XmaI restriction sites flanked by NheI/HindIII sites were inserted to replace the T7 promoter-DHFR-T7 terminator region, resulting in pIvi-MCS vector. Promoter sequences were obtained by PCR assembly and cloned into pIvi-MCS digested with $\mathrm{XbaI}$ and $\mathrm{XhoI}$. The pglpT is a strong constitutive promoter [18]. OXB20 is the strongest RecA promoter derivative with an ablated repressor binding site to enable constitutive expression (PSF-OXB20, OGS50, Sigma). The pJ23119 is the strongest promoter in a family of constitutive promoters isolated from a combinational library (PMID: 23560087). The pEm7 is a constitutive synthetic derivative of a T7 promoter (part Doulix biofundry, part ENW51Y). TagGFP2 and sfGFP genes were PCR amplified from pTagGFP2-N (Evrogen, Moscow, Russia) and pYTK047 plasmids, respectively, and cloned with XhoI/XmaI restriction sites. The pYTK047 was a gift from John Dueber (Addgene plasmid \# 65154; http:/ /n2t.net/addgene:65154; RRID:Addgene_65154, accessed on: 1 May 2018). The terminator region containing rrnB1 and T7 terminators was PCR amplified from pYTK047 and cloned with XmaI/HindIII restriction sites. The following E. coli strains were used: Rosetta (DE3) (Novagen, Madison, WI, USA), BL21(DE3) (Invitrogen, Waltham, MA, USA), TG1 (Lucigen, Middleton, WI, USA), XL-1 (Agilent, Santa Clara, CA, USA), and SHuffle T7 (NEB, Ipswich, MA, USA). Control GFP-producing reporter S. aureus cells were described previously [11-13].

Fluorescence measurements. E. coli cultures were grown overnight using 2YT medium (16 g/L tryptone, $10 \mathrm{~g} / \mathrm{L}$ yeast extract, $5 \mathrm{~g} / \mathrm{L} \mathrm{NaCl}$ ) supplemented with $100 \mu \mathrm{g} / \mathrm{mL}$ ampicillin in shaking flasks at $37^{\circ} \mathrm{C}$ and $250 \mathrm{rpm}$. Brain Heart Infusion (BHI) medium (BD, Franklin Lakes, NJ, USA) or TB medium (12 g/L tryptone, $24 \mathrm{~g} / \mathrm{L}$ yeast extract, $4 \mathrm{~g} / \mathrm{L}$ glycerol) was inoculated in 1:100 ratio and cultivated at $30^{\circ} \mathrm{C}$ for 1,2 , and 4 days. Fluorescence measurements were made using Varioskan Flash Multimode plate reader (Thermo Fisher Scientific, Waltham, MA, USA) with $\lambda_{\text {ex }} / \lambda_{\text {em }}=488 / 513 \mathrm{~nm}$ and NovoCyte Flow Cytometer (Agilent, Santa Clara, CA, USA). GFP-producing E. coli were visualized using an Eclipse Ti inverted fluorescence microscope (Nikon, Tokyo, Japan) with a standard FITC filter. Bacterial colonies grown on agar plates were analyzed by GFP fluorescence using VersaDoc (Bio-Rad, Hercules, CA, USA).

Encapsulation of $E$. coli in droplets. BL21(DE3) E. coli-producing sfGFP under control of pJ23119 promoter was cultured in BHI medium in shaking flasks at $37^{\circ} \mathrm{C}$ and $250 \mathrm{rpm}$ until early logarithmic growth phase. Subsequently, liquid cultures were filtered using 40- $\mu \mathrm{m}$ cell strainers (Greiner Bio-One) and 20- $\mu \mathrm{m}$ solvent filters (A-313, IDEX, Northbrook, $\mathrm{IL}, \mathrm{USA}$ ) and then diluted to reach $\mathrm{OD}_{600}=0.3$ (occupancy $(\lambda) \sim 5$ E. coli cells per a droplet). $E$. coli cells were encapsulated in droplets of microfluidic double emulsion (MDE), using 20- $\mu \mathrm{m}$ microfluidic chips produced via soft lithography, as was described previously [11]. MDE droplets with encapsulated bacterial cells were cultured at $30^{\circ} \mathrm{C}$ in a water vapor saturated incubator. MDE droplets were loaded into a hemocytometer and were visualized using an Eclipse Ti inverted fluorescence microscope (Nikon) with a standard FITC filter.

Ultrahigh-throughput screening of anti-Gram-negative antibiotic activity. The selection of bacteria displaying antibacterial activity was described in detail previously [11-13]. Briefly, sfGFP-producing E. coli cells were vitally stained with sulfo-Cyanine5 NHS (Lumiprobe, Moscow, Russia), washed, and filtered using 20- $\mu \mathrm{m}$ solvent filters (A-313, IDEX, Northbrook, IL, USA). The soil microbiome was isolated in the Moscow region. Microbiome samples were unfrozen directly before encapsulation, resuspended in BHI broth (BD, Franklin 
Lakes, NJ, USA), and filtered through 40- $\mu \mathrm{m}$ cell strainers (Greiner Bio-One, Monroe, NC, USA). The sfGFP-producing live biosensors were co-encapsulated with a soil microbiome suspension in droplets of MDE. After overnight incubation at $35^{\circ} \mathrm{C}$, Calcein Violet AM (Thermo Fisher Scientific, Waltham, MA, USA) was added to the droplet emulsion to the final concentration of $10 \mu \mathrm{M}$. Subsequently, the droplets with simultaneous $\mathrm{sCy} 5^{\text {high }}$, GFP ${ }^{\text {low }}$, and Calcein Violet ${ }^{\text {high }}$ fluorescence were sorted using a FACSAria III cell sorter (BD, Franklin Lakes, NJ, USA). Bacterial colonies were regenerated after plating on BHI-agar (BD, Franklin Lakes, NJ, USA) and tested for antibiotic activity against E. coli using the agar overlay assay.

P. polymyxa cultivation. P. polymyxa $\mathrm{P} 4$ was cultivated in SYC medium containing $40 \mathrm{~g} / \mathrm{L}$ sucrose, $5 \mathrm{~g} / \mathrm{L}$ yeast extract, $4 \mathrm{~g} / \mathrm{L} \mathrm{CaCO}_{3}, 1.5 \mathrm{~g} / \mathrm{L} \mathrm{K}_{2} \mathrm{HPO}_{4}, 2 \mathrm{~g} / \mathrm{L}$ glucose, $2 \mathrm{~g} / \mathrm{L}$ $\mathrm{NaCl}, 1.5 \mathrm{~g} / \mathrm{L} \mathrm{MgSO}_{4}, 2 \mathrm{~g} / \mathrm{L}\left(\mathrm{NH}_{4}\right)_{2} \mathrm{SO}_{4}, 0.01 \mathrm{~g} / \mathrm{L} \mathrm{FeSO}_{4}$, and $0.01 \mathrm{~g} / \mathrm{L} \mathrm{MnCl}_{2}$ for $24 \mathrm{~h}$ at $30^{\circ} \mathrm{C}$. P. polymyxa $\mathrm{P} 4$ was inoculated from overnight culture using 1:100 dilution and cultivated using 750-mL flasks in $100 \mathrm{~mL}$ with $250 \mathrm{rpm}$ shaking for 4 days.

Antimicrobial activity. Inhibition of bacterial cell growth was measured by a doubling dilution of P. polymyxa P4 medium and C18 HPLC fractions in 2YT medium inoculated with $E$. coli $\mathrm{OD}_{600}=0.002$. After overnight incubation at $30{ }^{\circ} \mathrm{C}$, E. coli growth was analyzed by GFP fluorescence $\left(\lambda_{\mathrm{ex}} / \lambda_{\mathrm{em}}=488 / 513 \mathrm{~nm}\right)$ and $\mathrm{OD}_{600}$ using a Varioskan Flash Multimode Reader (Thermo Fisher Scientific).

Whole-genome sequencing and bioinformatic analysis. Total DNA was isolated using the QIAamp DNA Investigator Kit (Qiagen, Germantown, MD, USA). Genomic DNA was disrupted into 400-550-bp fragments by Covaris S220 System (Covaris, Woburn, MA, USA). Fragment libraries were prepared using the NEBNext ${ }^{\circledR}$ DNA Library Prep Reagent Set for Illumina and the NEBNext ${ }^{\circledR}$ Multiplex Oligos for Illumina ${ }^{\circledR}$ (96 Index Primers) (Illumina, San Diego, CA, USA) according to the manufacturer's instructions. Sequencing of libraries was performed using the genetic analyzer HiSeq2500, the HiSeq ${ }^{\circledR}$ PE Cluster Kit v4-cBot ${ }^{\mathrm{TM}}$, and the HiSeq ${ }^{\circledR}$ SBS Kit v4 (250 cycles) (Illumina, San Diego, CA, USA) according to the manufacturer's instructions. Genome assemblies were performed using SPAdes 3.9.0 [29]. Genomes were annotated with prokka [30]. Identification of biosynthetic gene clusters and NRPS modular organization was performed with antiSMASH 6.0 [31]. Comparative analysis of homologous gene clusters was provided by MultiGeneBlast [32] based on the MIBiG database [33].

Active metabolite extraction and metabolomic analysis. The culture medium of P. polymyxa P4 was centrifuged at $10,000 \times g$ for $10 \mathrm{~min}$. Active metabolites were extracted by solid-phase extraction with LPS-500 sorbent (Technosorbent, Moscow, Russia) using buffer $\mathrm{A}\left(10 \mathrm{mM} \mathrm{NH}_{4} \mathrm{OAc} \mathrm{pH} 6.0,20 \% \mathrm{ACN}\right)$ for sorbent wash and buffer $\mathrm{B}(10 \mathrm{mM}$ $\mathrm{NH}_{4} \mathrm{OAc} \mathrm{pH} 6.0,80 \% \mathrm{ACN}$ ) for elution. LC-MS analysis was carried out on an Ultimate 3000 RSLCnano HPLC system connected to an Orbitrap Fusion Lumos mass spectrometer (ThermoFisher Scientific) with the loading pump used for analytical flow gradient delivery. Samples were separated on Luna Omega C18 $1.6 \mu \mathrm{m} 100 \AA$ column $100 \times 2.1 \mathrm{~mm}$ at a $200 \mu \mathrm{L} / \mathrm{min}$ flow rate. Separation was done by a gradient of $99.9 \% \mathrm{ACN}, 10 \mathrm{mM}$ ammonium formate, $0.1 \%$ FA (Buffer B) in $99.9 \% \mathrm{H}_{2} \mathrm{O}, 10 \mathrm{mM}$ ammonium formate, $0.1 \%$ FA (Buffer A): $5 \%$ B at $0 \mathrm{~min}, 5 \% \mathrm{~B}$ at $5 \mathrm{~min}, 99 \% \mathrm{~B}$ at $20 \mathrm{~min}$, followed by $5 \mathrm{~min}$ wash at $99 \% \mathrm{~B}$ and 10 min equilibration at $5 \%$ B before the next run. UV data were collected at 260 and $315 \mathrm{~nm}$. MS1 spectra were collected in Positive ion mode at $30 \mathrm{~K}$ Orbitrap resolution in profile mode with 200-2000 a.e.m mass range and RF lens 30\%. For the rest of the MS1 parameters as well as for the ESI parameters, the default values suggested by Xcalibur software ver. 4.3.73.11 were taken. MS2 precursors were selected based on MS1 intensity: Intensity threshold was $5 \times 10^{4}$ with the dynamic exclusion set to $10 \mathrm{~s}$ after two selections with the mass tolerance of $10 \mathrm{ppm}$ and isotope exclusion. MS2 spectra were collected at $15 \mathrm{~K}$ resolution in centroid mode. The isolation window was set to $1 / 6 \mathrm{~m} / \mathrm{z}$ with no offset and Quadrupole isolation mode. Fragmentation was done by HCD with a stepped CE of 20,35 , and $50 \%$. The rest of the MS2 parameters were taken as default values. The total MS1-MS2 cycle time was selected to $1 \mathrm{sec}$. LC-MS/MS raw data were analyzed in Compound Discoverer 3.2 (Thermo Fisher Scientific). Peak annotation was performed with 
ChemSpider, Natural Product Atlas 2020, and MzCloud databases using MS1 and MS1MS2 information correspondingly with $5 \mathrm{ppm}$ mass accuracy, isotopic distribution $\geq 50 \%$, and match score $\geq 85 \%$.

Author Contributions: M.N.B., I.V.S., A.G.G. and S.S.T. designed the research; M.N.B., P.A.B., A.M.K., Y.A.M., O.A.B. and M.A.Y. performed the research; O.A.B. and S.K. contributed new analytic tools; M.N.B., S.K., Y.A.M., S.S.T., I.V.S. and A.G.G. analyzed the data; M.N.B., I.V.S., A.G.G. and S.S.T. wrote the paper. All authors have read and agreed to the published version of the manuscript.

Funding: This work was supported by Grant 21-14-00357 from the Russian Science Foundation and personal scholarships from the Council for Grants of the President of the Russian Federation СП-3370.2019.4 (Y.A.M.) and СП-2911.2019.4 (S.S.T.).

Institutional Review Board Statement: Not applicable.

Informed Consent Statement: Not applicable.

Data Availability Statement: Data is contained within the article. Additional data are freely available on request from the corresponding author.

Conflicts of Interest: The authors declare no conflict of interest.

\section{References}

1. Fleischmann, M.C.; Scherag, A.; Adhikari, N.K.J.; Hartog, C.S.; Tsaganos, T.; Schlattmann, P.; Angus, D.C.; Reinhart, K. Assessment of Global Incidence and Mortality of Hospital-treated Sepsis. Current Estimates and Limitations. Am. J. Respir. Crit. Care Med. 2016, 193, 259-272. [CrossRef]

2. Rudd, K.E.; Johnson, S.C.; Agesa, K.M.; Shackelford, K.A.; Tsoi, D.; Kievlan, D.R.; Colombara, D.V.; Ikuta, K.S.; Kissoon, N.; Finfer, S.; et al. Global, regional, and national sepsis incidence and mortality, 1990-2017: Analysis for the Global Burden of Disease Study. Lancet 2020, 395, 200-211. [CrossRef]

3. Sulis, G.; Batomen, B.; Kotwani, A.; Pai, M.; Gandra, S. Sales of antibiotics and hydroxychloroquine in India during the COVID-19 epidemic: An interrupted time series analysis. PLoS Med. 2021, 18, e1003682. [CrossRef] [PubMed]

4. Elsayed, A.A.; Darwish, S.F.; Zewail, M.B.; Mohammed, M.; Saeed, H.; Rabea, H. Antibiotic misuse and compliance with infection control measures during COVID-19 pandemic in community pharmacies in Egypt. Int. J. Clin. Pract. 2021, 75, e14081. [CrossRef]

5. Centers for Disease Control and Prevention. Antibiotic Resistance Threats in the United States. 2019. Available online: https: / / www.cdc.gov / drugresistance/pdf/threatsreport/2019-ar-threats-report-508.pdf (accessed on 21 February 2020).

6. Fitzpatrick, M.A. Real-world antibiotic needs for resistant Gram-negative infections. Lancet Infect. Dis. 2020, 20, 1108-1109. [CrossRef]

7. Breijyeh, Z.; Jubeh, B.; Karaman, R. Resistance of Gram-Negative Bacteria to Current Antibacterial Agents and Approaches to Resolve It. Molecules 2020, 25, 1340. [CrossRef] [PubMed]

8. Culp, E.J.; Waglechner, N.; Wang, W.; Fiebig-Comyn, A.A.; Hsu, Y.-P.; Koteva, K.; Sychantha, D.; Coombes, B.K.; Van Nieuwenhze, M.S.; Brun, Y.V.; et al. Evolution-guided discovery of antibiotics that inhibit peptidoglycan remodelling. Nat. Cell Biol. 2020, 578, 582-587. [CrossRef]

9. Genilloud, O. Actinomycetes: Still a source of novel antibiotics. Nat. Prod. Rep. 2017, 34, 1203-1232. [CrossRef]

10. Imai, Y.; Meyer, K.J.; Iinishi, A.; Favre-Godal, Q.; Green, R.; Manuse, S.; Caboni, M.; Mori, M.; Niles, S.; Ghiglieri, M.; et al. A new antibiotic selectively kills Gram-negative pathogens. Nature 2019, 576, 459-464. [CrossRef]

11. Terekhov, S.; Smirnov, I.; Stepanova, A.V.; Bobik, T.V.; Mokrushina, Y.; Ponomarenko, N.A.; Belogurov, A.A., Jr.; Rubtsova, M.P.; Kartseva, O.; Gomzikova, M.O.; et al. Microfluidic droplet platform for ultrahigh-throughput single-cell screening of biodiversity. Proc. Natl. Acad. Sci. USA 2017, 114, 2550-2555. [CrossRef]

12. Terekhov, S.; Smirnov, I.; Malakhova, M.V.; Samoilov, A.; Manolov, A.I.; Nazarov, A.S.; Danilov, D.V.; Dubiley, S.A.; Osterman, I.; Rubtsova, M.P.; et al. Ultrahigh-throughput functional profiling of microbiota communities. Proc. Natl. Acad. Sci. USA 2018, 115, 9551-9556. [CrossRef]

13. Terekhov, S.S.; Nazarov, A.S.; Mokrushina, Y.A.; Baranova, M.N.; Potapova, N.A.; Malakhova, M.V.; Ilina, E.N.; Smirnov, I.V.; Gabibov, A.G. Deep Functional Profiling Facilitates the Evaluation of the Antibacterial Potential of the Antibiotic Amicoumacin. Antibiotics 2020, 9, 157. [CrossRef] [PubMed]

14. Terekhov, S.S.; Mokrushina, Y.A.; Nazarov, A.S.; Zlobin, A.; Zalevsky, A.; Bourenkov, G.; Golovin, A.; Belogurov, A., Jr.; Osterman, I.A.; Kulikova, A.A.; et al. A kinase bioscavenger provides antibiotic resistance by extremely tight substrate binding. Sci. Adv. 2020, 6, eaaz9861. [CrossRef] [PubMed]

15. Subach, O.M.; Gundorov, I.S.; Yoshimura, M.; Subach, F.V.; Zhang, J.; Grüenwald, D.; Souslova, E.A.; Chudakov, D.; Verkhusha, V.V. Conversion of Red Fluorescent Protein into a Bright Blue Probe. Chem. Biol. 2008, 15, 1116-1124. [CrossRef] [PubMed]

16. Pédelacq, J.-D.; Cabantous, S.; Tran, T.; Terwilliger, T.; Waldo, G.S. Engineering and characterization of a superfolder green fluorescent protein. Nat. Biotechnol. 2005, 24, 79-88. [CrossRef] [PubMed] 
17. Lane, M.C.; Alteri, C.J.; Smith, S.N.; Mobley, H.L.T. Expression of flagella is coincident with uropathogenic Escherichia coli ascension to the upper urinary tract. Proc. Natl. Acad. Sci. USA 2007, 104, 16669-16674. [CrossRef] [PubMed]

18. Liu, X.; Yuk, H.; Lin, S.; Parada, G.A.; Tang, T.-C.; Tham, E.; de la Fuente-Nunez, C.; Lu, T.K.; Zhao, X. 3D Printing of Living Responsive Materials and Devices. Adv. Mater. 2018, 30, 1704821. [CrossRef] [PubMed]

19. Yan, Q.; Fong, S.S. Study of in vitro transcriptional binding effects and noise using constitutive promoters combined with UP element sequences in Escherichia coli. J. Biol. Eng. 2017, 11, 33. [CrossRef]

20. Zhu, Y.; Hua, Y.; Zhang, B.; Sun, L.; Li, W.; Kong, X.; Hong, J. Metabolic engineering of indole pyruvic acid biosynthesis in Escherichia coli with tdiD. Microb. Cell Factories 2017, 16, 1-15. [CrossRef]

21. Choi, S.-K.; Park, S.-Y.; Kim, R.; Kim, S.-B.; Lee, C.-H.; Kim, J.F.; Park, S.-H. Identification of a Polymyxin Synthetase Gene Cluster of Paenibacillus polymyxa and Heterologous Expression of the Gene in Bacillus subtilis. J. Bacteriol. 2009, 191, $3350-3358$. [CrossRef]

22. Shaheen, M.; Li, J.; Ross, A.C.; Vederas, J.; Jensen, S.E. Paenibacillus polymyxa PKB1 Produces Variants of Polymyxin B-Type Antibiotics. Chem. Biol. 2011, 18, 1640-1648. [CrossRef] [PubMed]

23. Tambadou, F.; Caradec, T.; Gagez, A.-L.; Bonnet, A.; Sopéna, V.; Bridiau, N.; Thiery, V.; Didelot, S.; Barthélémy, C.; Chevrot, R. Characterization of the colistin (polymyxin E1 and E2) biosynthetic gene cluster. Arch. Microbiol. 2015, 197, 521-532. [CrossRef] [PubMed]

24. Lee, M.E.; DeLoache, W.C.; Cervantes, B.; Dueber, J.E. A Highly Characterized Yeast Toolkit for Modular, Multipart Assembly. ACS Synth. Biol. 2015, 4, 975-986. [CrossRef]

25. Baltz, R.H. Renaissance in antibacterial discovery from actinomycetes. Curr. Opin. Pharmacol. 2008, 8, 557-563. [CrossRef] [PubMed]

26. Watterson, W.J.; Tanyeri, M.; Watson, A.R.; Cham, C.M.; Shan, Y.; Chang, E.B.; Eren, A.M.; Tay, S. Droplet-based high-throughput cultivation for accurate screening of antibiotic resistant gut microbes. eLife 2020, 9. [CrossRef]

27. Saito, K.; Ota, Y.; Tourlousse, D.M.; Matsukura, S.; Fujitani, H.; Morita, M.; Tsuneda, S.; Noda, N. Microdroplet-based system for culturing of environmental microorganisms using FNAP-sort. Sci. Rep. 2021, 11, 1-9. [CrossRef]

28. Mahler, L.; Niehs, S.P.; Martin, K.; Weber, T.; Scherlach, K.; Hertweck, C.; Roth, M.; Rosenbaum, M.A. Highly parallelized droplet cultivation and prioritization of antibiotic producers from natural microbial communities. eLife 2021, 10, 64774. [CrossRef]

29. Bankevich, A.; Nurk, S.; Antipov, D.; Gurevich, A.A.; Dvorkin, M.; Kulikov, A.S.; Lesin, V.M.; Nikolenko, S.I.; Pham, S.; Prjibelski, A.D.; et al. SPAdes: A New Genome Assembly Algorithm and Its Applications to Single-Cell Sequencing. J. Comput. Biol. 2012, 19, 455-477. [CrossRef]

30. Seemann, T. Prokka: Rapid Prokaryotic Genome Annotation. Bioinformatics 2014, 30, 2068-2069. [CrossRef]

31. Blin, K.; Shaw, S.; Kloosterman, A.M.; Charlop-Powers, Z.; van Wezel, G.P.; Medema, M.H.; Weber, T. antiSMASH 6.0: Improving cluster detection and comparison capabilities. Nucleic Acids Res. 2021, 49, W29-W35. [CrossRef] [PubMed]

32. Medema, M.H.; Takano, E.; Breitling, R. Detecting Sequence Homology at the Gene Cluster Level with MultiGeneBlast. Mol. Biol. Evol. 2013, 30, 1218-1223. [CrossRef] [PubMed]

33. Kautsar, S.A.; Blin, K.; Shaw, S.; Navarro-Muñoz, J.C.; Terlouw, B.R.; Van Der Hooft, J.J.J.; Van Santen, J.A.; Tracanna, V.; Duran, H.G.S.; Andreu, V.P.; et al. MIBiG 2.0: A repository for biosynthetic gene clusters of known function. Nucleic Acids Res. 2019, 48, D454-D458. [CrossRef] [PubMed] 